Supporting information for

\title{
A Novel Redox Modulator Induces a GPX4-mediated Cell Death That Is Dependent on Iron and Reactive Oxygen Species
}

Shuai Hu, ${ }^{\text {a b }}$ Mario Sechi,,${ }^{c}{ }^{*}$ Pankaj Kumar Singh, ${ }^{c}$ Lipeng Dai, ${ }^{d}$ Sean McCann, ${ }^{a}$ Duxin Sun, ${ }^{d}$ Mats Ljungman, ${ }^{\mathrm{e}}$ Nouri Neamati a, *

Figure S1. Characterization of originally synthesized QD394 analogs. ........................S3

Figure S2. QD394 induced a bit more ROS than QD325 in MIA PaCa-2 cells..............S3

Figure S3. QD394 did not change GSH level significantly, but napabucasin and BSO did.

Figure S4. Combination studies between QD394, napabucasin, and cell death inhibitors in MIA PaCa-2 cells. .S5

Figure S5. QD394 and QD394-Me reduced the melting temperature of GPX4 in PANC-1 cells.

Figure S6. Combination of NAC, dicoumarol (DIC) with QD394, QD394-Me, napabucasin (napa) and $\mathrm{H} 2 \mathrm{O} 2$ in probing LRPPRC and PNPT1 in pancreatic cancer cells.

Figure S7. Combination of QD394 and napabucasin in PANC-1 cells.

.S6

Figure S8. Full colony formation assay image of combination studies between QD394 and select drugs, and the synegism calculation using HSA and Bliss models from the Combenefit software.

Figure S9. Plasma concentration of QD394 and QD394-Me administered via IP, PO, or IV in CD-1 mice. S8

Figure S10. Major metabolite of QD394-Me in mouse plasma and its abundance profiles administered via IV or PO in mice. S8

Figure S11. In vivo study with female Balb/c mice implanted subcutaneously with CT-26 cells. S8

Figure S12. HPLC of lead compounds, (A) QD394 and (B) QD394-Me.......................S9 Table S1. Elemental analyses for compounds QD385-395, and QD394-Me (QD430)....S9 
Table S2. Molecular formula strings of compounds.

$\mathrm{S} 10$

Table S3. Top 25 upregulated (A) and downregulated (B) enriched C2 gene sets in QD394-treated MIA PaCa-2 cells. FDR $<0.001$. S10

Table S4. STRING analysis of 17 upregulated genes/proteins in common between Bru-seq and proteomics. Biological process GO terms are ranked by FDR. S12

Table S5. STRING analysis of 35 downregulated genes/proteins in common between Bru-seq and proteomics. Biological process GO terms are ranked by FDR. PNPT1 and LRPPRC are labeled in red. S13

Table S6. Tissue distribution of QD394-Me in mouse. IV, $10 \mathrm{mg} / \mathrm{kg}$; PO, $20 \mathrm{mg} / \mathrm{kg}, 1 \mathrm{~h}$. S15

Table S7. Cytotoxicity of QD394, QD394-Me, and napabucasin in GPX4-knockdown PANC-1 and BxPC-3 cells. siNC: samples treated with scramble siRNA; siGPX4: samples treated with GPX4 siRNA.

Table S8. Top upregulated (A) and downregulated (B) enriched hallmark gene sets in QD394-Me-treated MIA PaCa-2 cells. FDR $<0.05$. S 15

Table S9. Top upregulated enriched KEGG gene sets in QD394-Me-treated MIA PaCa-2 cells. No downregulated enriched KEGG gene sets were significant. FDR $<0.05$.......S16 

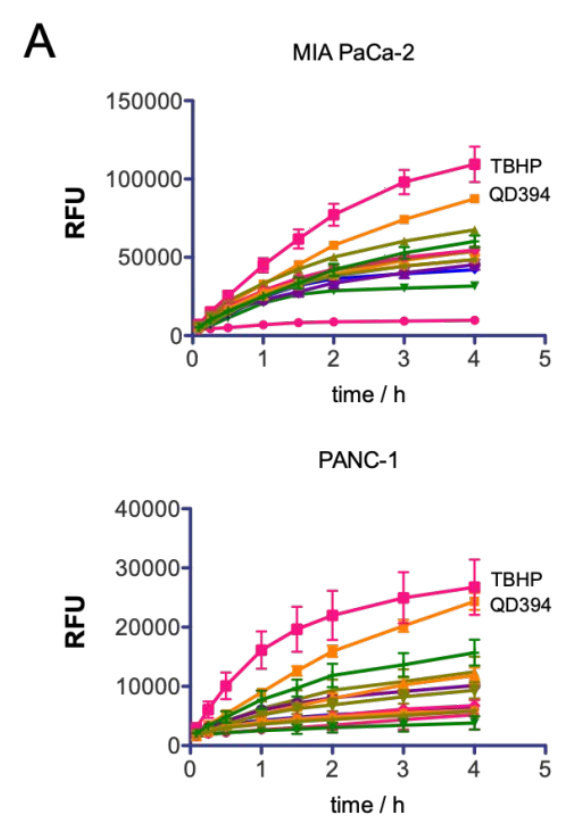

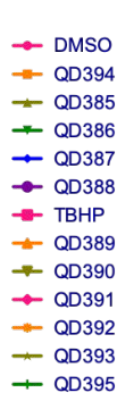

$\leadsto$ DMSO

- DMSO
- QD394

-QD385

- QD386

$\rightarrow$ QD387

- QD388
- TBHP

- QD389

- QD389
- QD390

$\rightarrow$ QD391

- QD392

- QD393
+ QD395

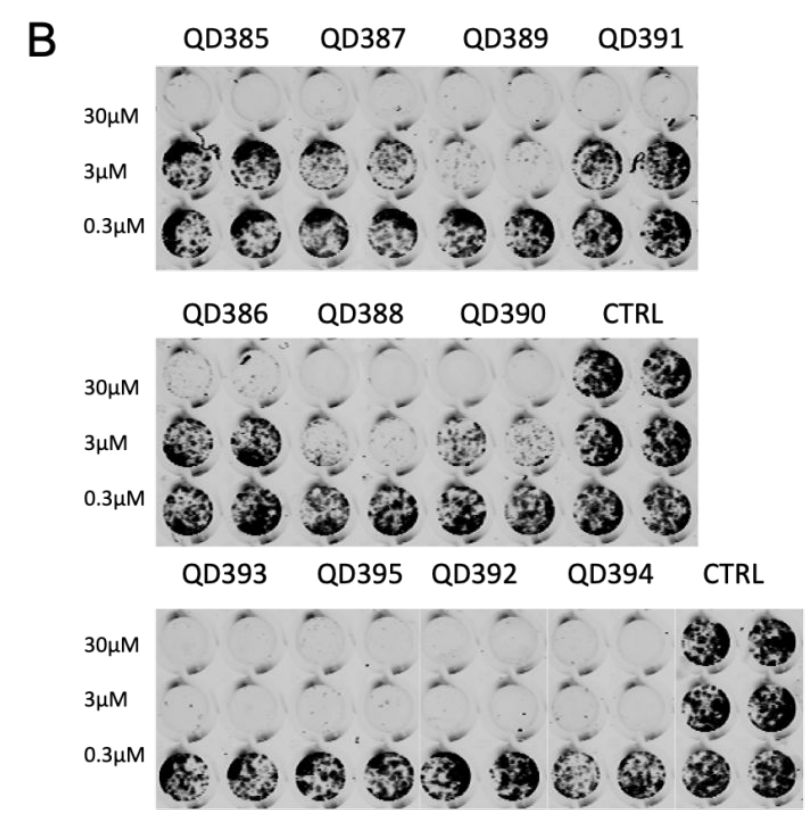

Figure S1. Characterization of originally synthesized QD394 analogs.

(A) ROS-inducing capability of 11 QD394 analogs measured by CM-H2DCFDA dye in MIA PaCa-2 and PANC-1 cells. RFU, relative fluorescence unit. All analogs were tested at $10 \mu \mathrm{M}$, and TBHP was tested at $200 \mu \mathrm{M}$. (B) QD394 is one of the most potent compounds in the colony formation assay in MIA PaCa-2 cells.
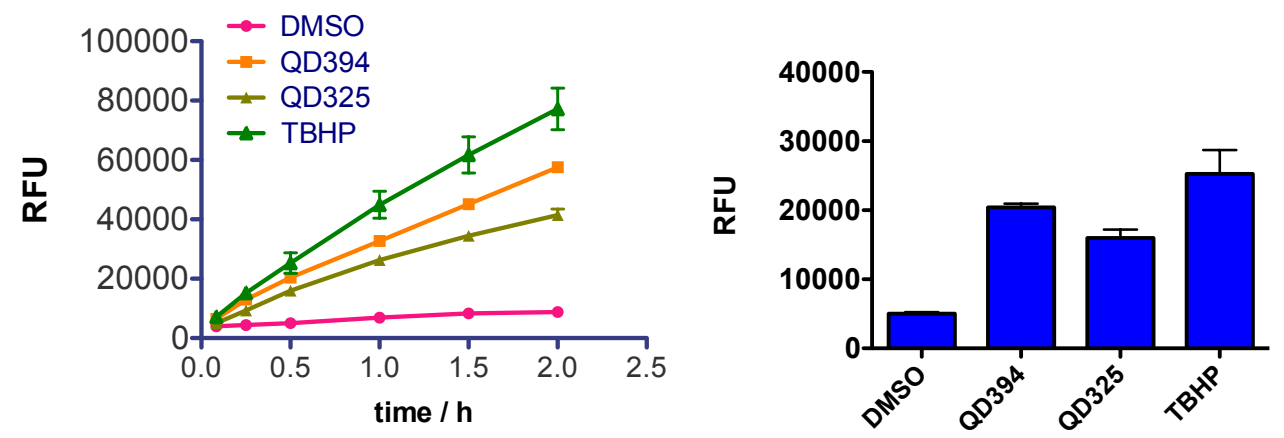

Figure S2. QD394 induced a bit more ROS than QD325 in MIA PaCa-2 cells.

$\mathrm{RFU}$, relative fluorescence unit. Compounds were tested at $10 \mu \mathrm{M}$, and TBHP was tested at $200 \mu \mathrm{M}$. Barplot was made using data at $30 \mathrm{~min}$. 


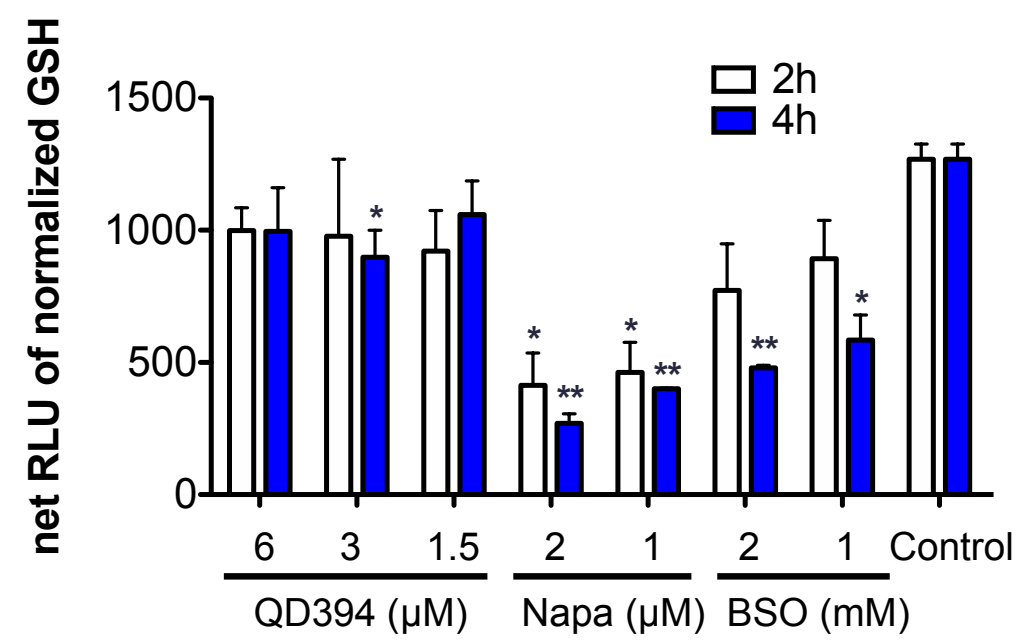

Figure S3. QD394 did not change GSH level significantly, but napabucasin and BSO did. RLU, relative luminescence unit. * denotes $\mathrm{p}<0.05, * *$ denotes $\mathrm{p}<0.005, * * *$ denotes $\mathrm{p}$ $<0.0005$.
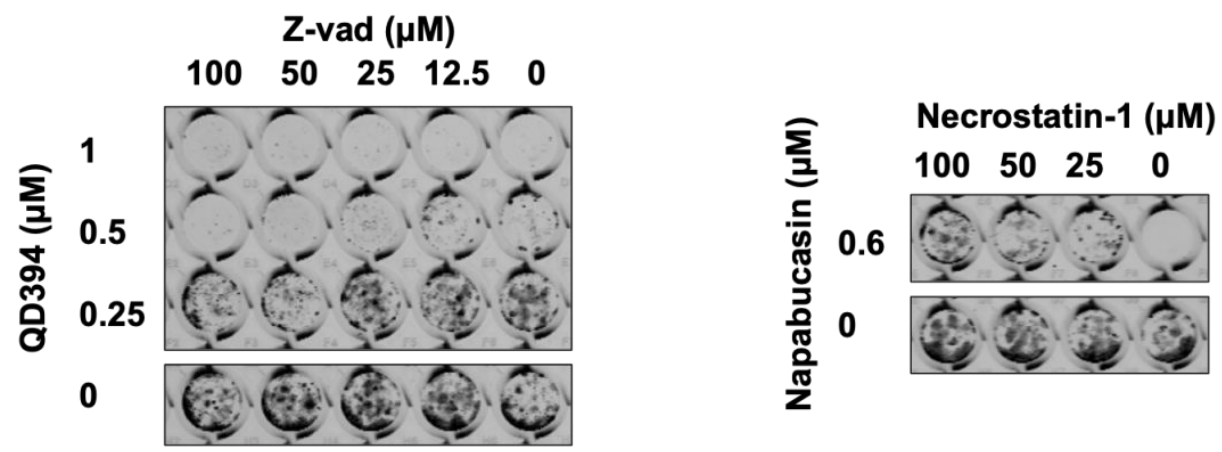

Necrostatin-1 $(\mu \mathrm{M}) \quad$ Chloroquine $(\mu \mathrm{M})$
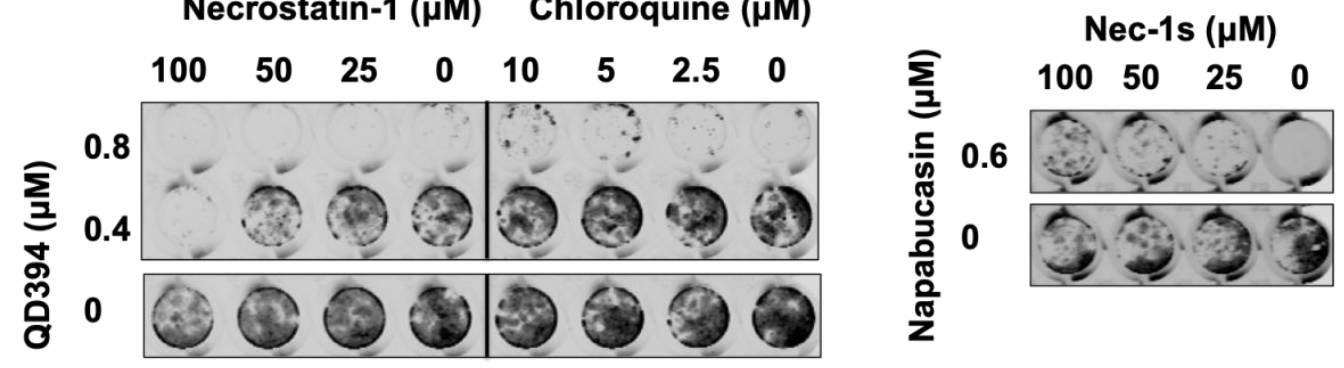

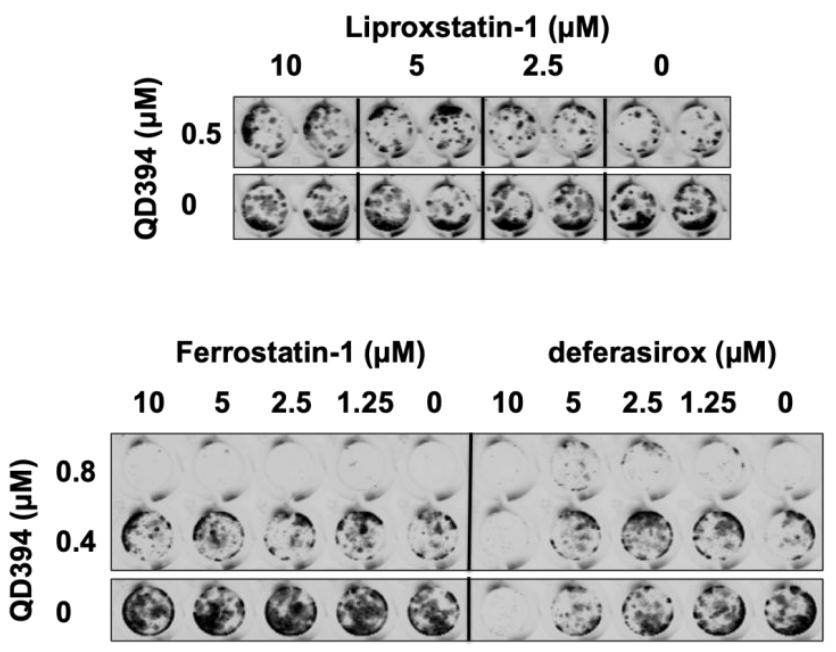

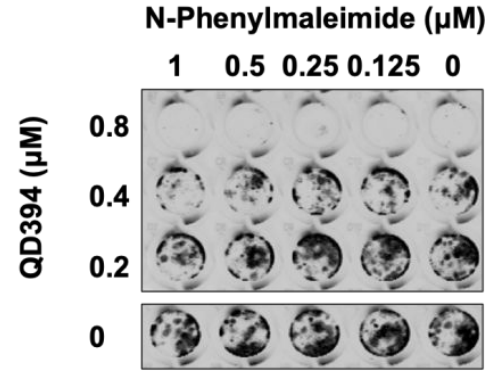

3-Methyladenine ( $\mathrm{mM})$ 2.51 .250 .6250 .3130

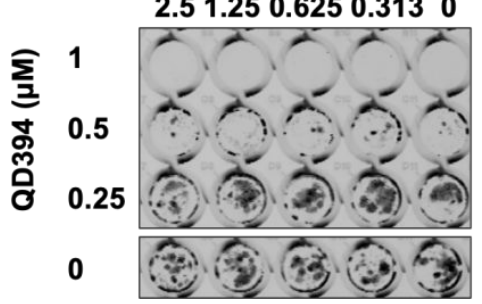

Figure S4. Combination studies between QD394, napabucasin, and cell death inhibitors in MIA PaCa-2 cells.

A

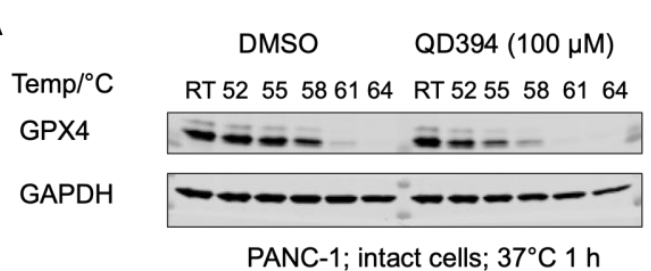

B

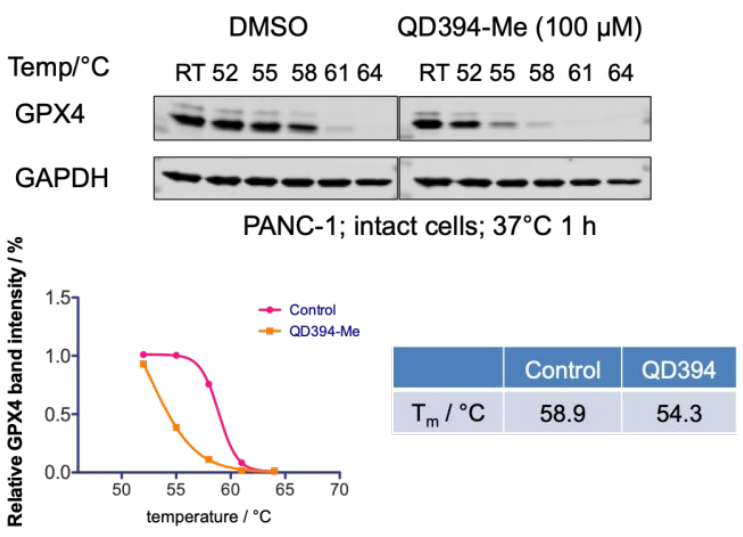

Figure S5. QD394 and QD394-Me reduced the melting temperature of GPX4 in PANC-1 cells.

(A) QD394. (B) QD394-Me. RT: around $21^{\circ} \mathrm{C}$. 
A

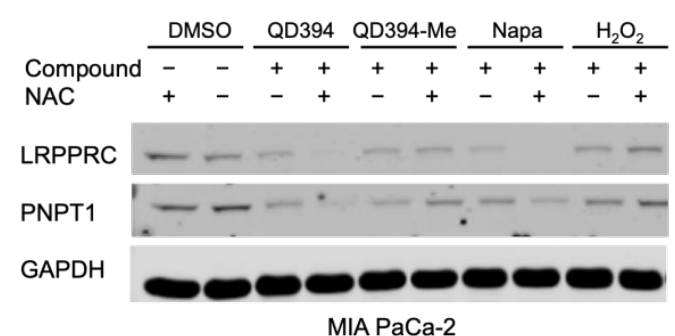

MIA PaCa-2
B

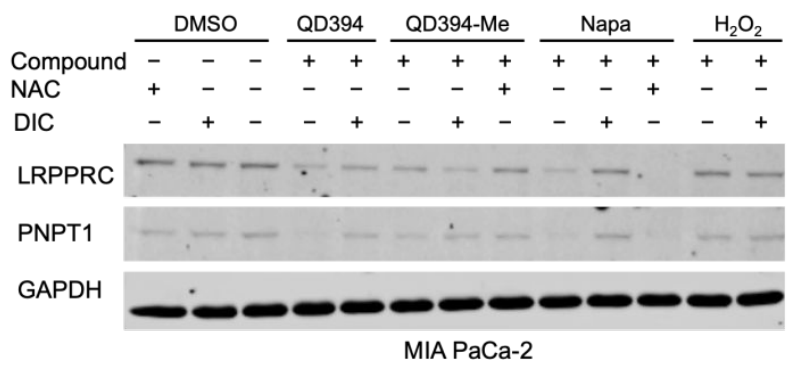

C DMSO $\frac{\mathrm{QD} 394}{1 \mathrm{~h} 4 \mathrm{~h} 24 \mathrm{~h}} \frac{\mathrm{QD} 394-\mathrm{Me}}{1 \mathrm{~h} 4 \mathrm{~h} 24 \mathrm{~h}} \frac{\mathrm{Napa}}{1 \mathrm{~h} 4 \mathrm{~h} 24 \mathrm{~h}} \frac{\mathrm{H}_{2} \mathrm{O}_{2}}{1 \mathrm{~h} 4 \mathrm{~h} 24 \mathrm{~h}}$ LRPPRC - - - - - - - - - - - PNPT1 GAPDH $---\div-\div-\div-1-1$ PANC-1 BxPC-3

Figure S6. Combination of NAC, dicoumarol (DIC) with QD394, QD394-Me, napabucasin (napa) and $\mathrm{H} 2 \mathrm{O} 2$ in probing LRPPRC and PNPT1 in pancreatic cancer cells. QD394, QD394-Me and napabucasin were tested at $3 \times$ IC50 for $4 \mathrm{~h}$. H2O2 was tested at $200 \mu \mathrm{M}$. NAC $(3 \mathrm{mM})$ and DIC $(20 \mu \mathrm{M})$ were added $1 \mathrm{~h}$ before compound treatments. (A, B) MIA PaCa-2. (C) PANC-1. (D) BxPC-3.

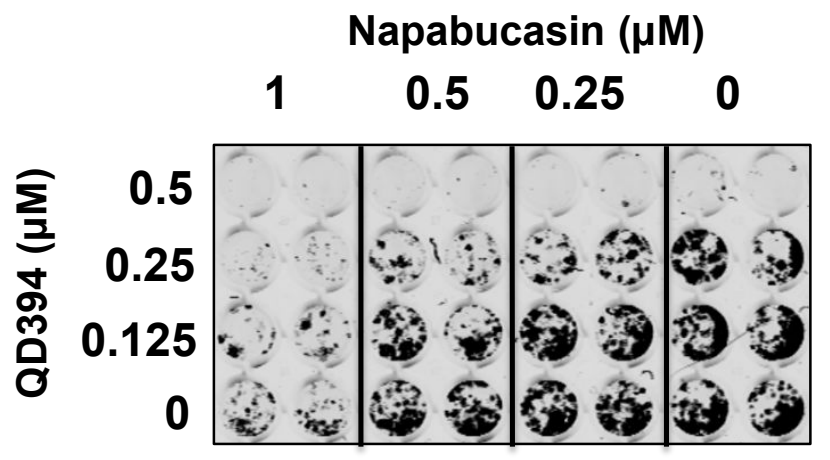

Figure S7. Combination of QD394 and napabucasin in PANC-1 cells. 

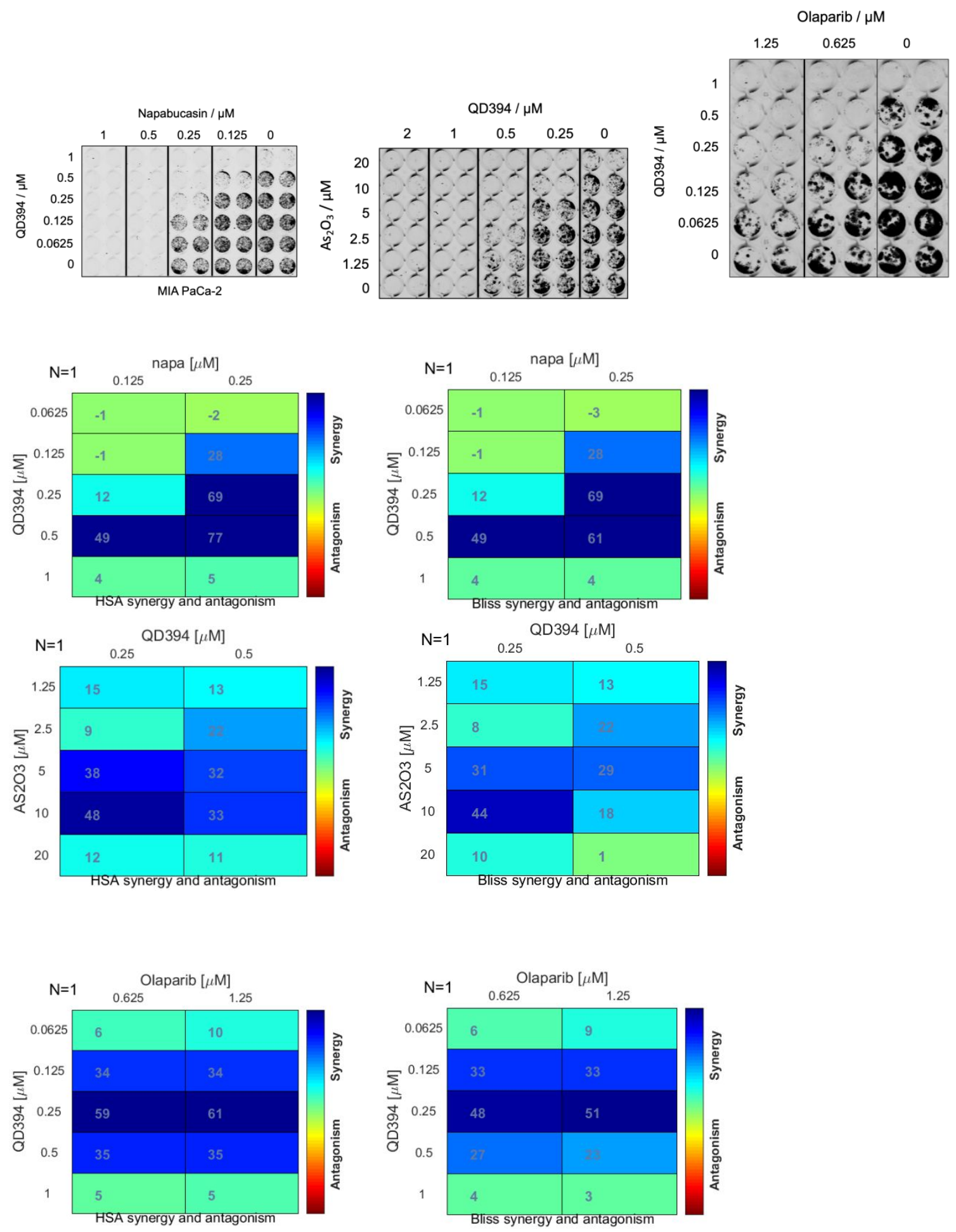

Figure S8. Full colony formation assay image of combination studies between QD394 and select drugs, and the synegism calculation using HSA and Bliss models from the Combenefit software. 

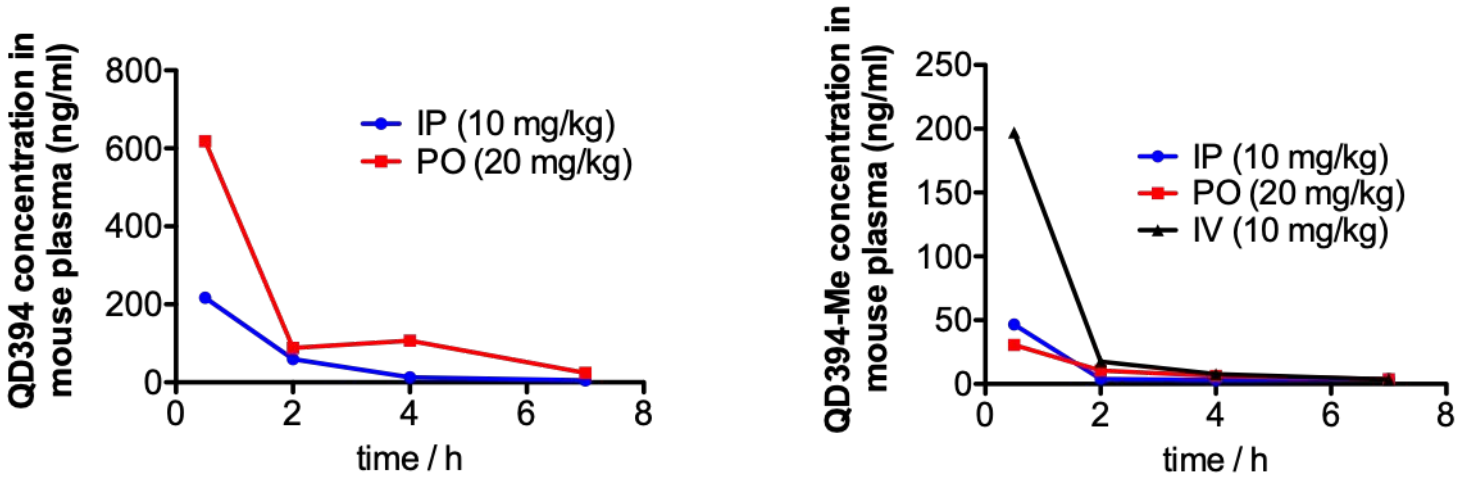

Figure S9. Plasma concentration of QD394 and QD394-Me administered via IP, PO, or IV in CD-1 mice.
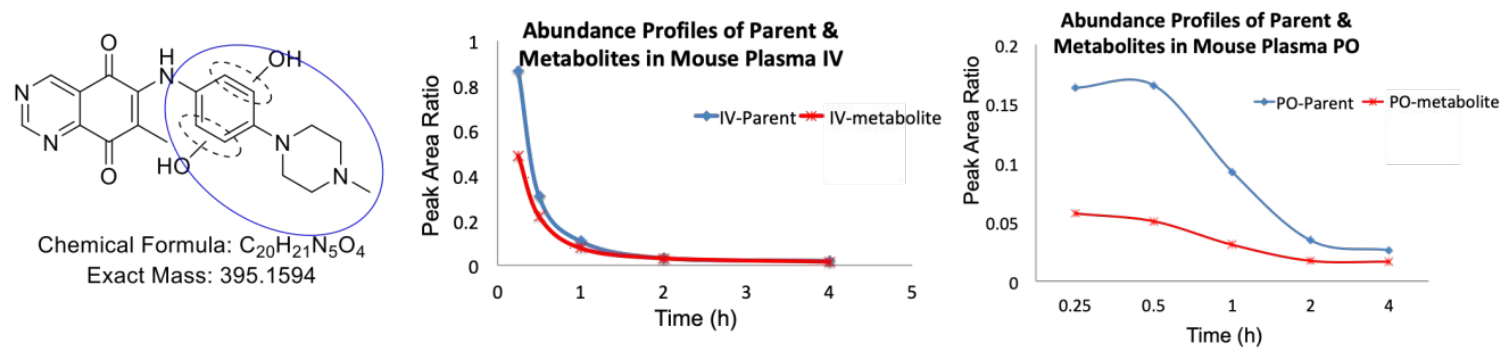

Figure S10. Major metabolite of QD394-Me in mouse plasma and its abundance profiles administered via IV or PO in mice.

Two hydroxyl groups could be on either ring within the circle.
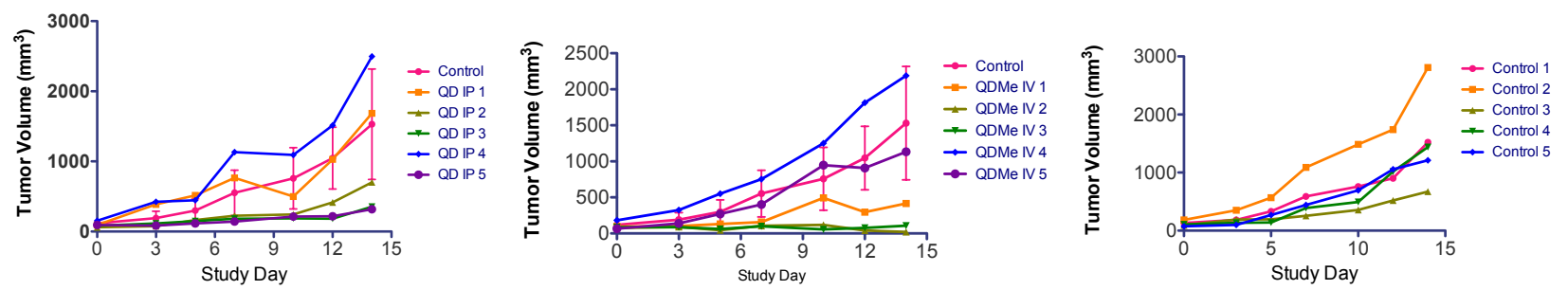

Figure S11. In vivo study with female Balb/c mice implanted subcutaneously with CT-26 cells.

QD394 (QD) was dosed at $10 \mathrm{mg} / \mathrm{kg}$ IP, and QD394-Me (QDMe) was dosed 3 times weekly at $20 \mathrm{mg} / \mathrm{kg} \mathrm{IV}$. Five mice were in each group. 
Figure S12. HPLC of lead compounds, (A) QD394 and (B) QD394-Me.

(A)

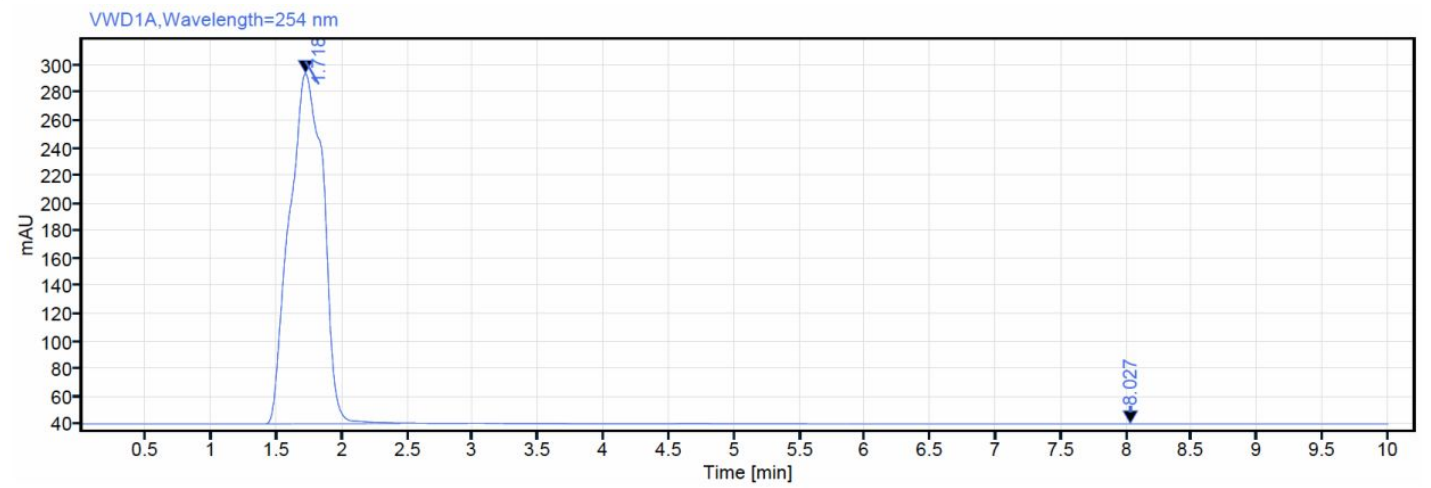

(B)

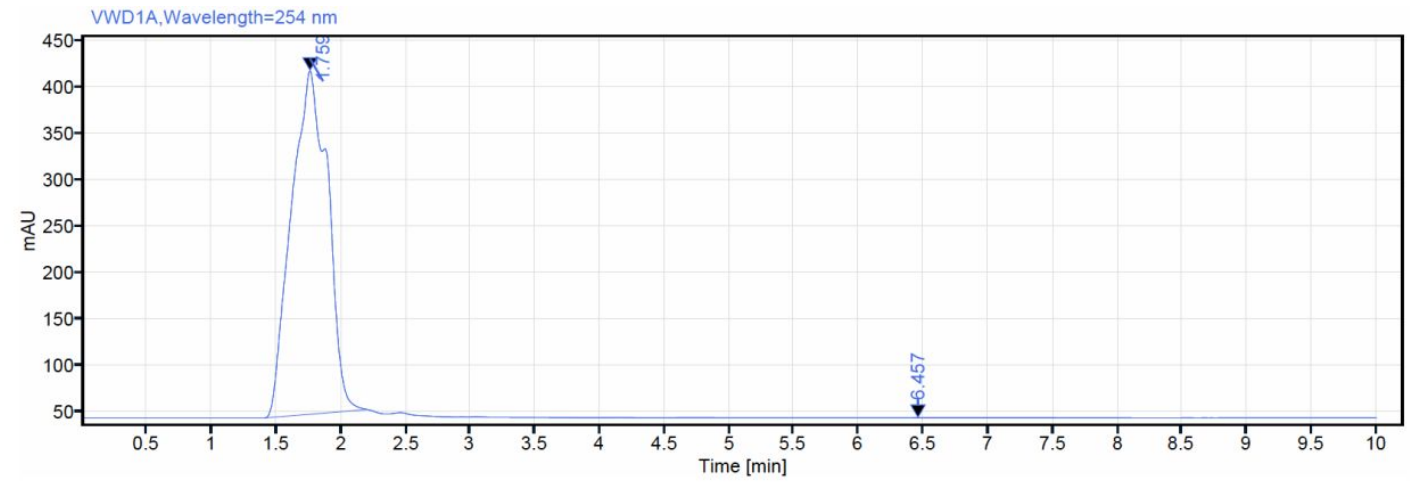

Table S1. Elemental analyses for compounds QD385-395, and QD394-Me (QD430).

\begin{tabular}{llllllll}
\hline \multirow{2}{*}{ Compound } & \multirow{2}{*}{ Molecular Formula } & \multicolumn{3}{c}{ Calculated } & \multicolumn{3}{c}{ Found } \\
& & $\% \mathrm{C}$ & $\% \mathrm{H}$ & $\mathrm{N}$ & $\% \mathrm{C}$ & $\% \mathrm{H}$ & $\% \mathrm{~N}$ \\
\hline QD385 & $\mathrm{C}_{20} \mathrm{H}_{13} \mathrm{~N}_{3} \mathrm{O}_{2}$ & 73.38 & 4.00 & 12.84 & 73.54 & 3.79 & 12.97 \\
QD386 & $\mathrm{C}_{20} \mathrm{H}_{12} \mathrm{ClN}_{3} \mathrm{O}_{2}$ & 66.40 & 3.34 & 11.61 & 66.27 & 3.30 & 11.72 \\
QD387 & $\mathrm{C}_{20} \mathrm{H}_{11} \mathrm{Cl}_{2} \mathrm{~N}_{3} \mathrm{O}_{2}$ & 60.63 & 2.80 & 10.61 & 60.71 & 2.64 & 10.44 \\
QD388 & $\mathrm{C}_{19} \mathrm{H}_{18} \mathrm{~N}_{4} \mathrm{O}_{2}$ & 68.25 & 5.43 & 16.76 & 68.06 & 5.56 & 16.58 \\
QD389 & $\mathrm{C}_{18} \mathrm{H}_{16} \mathrm{~N}_{4} \mathrm{O}_{3}$ & 64.28 & 4.79 & 12.66 & 64.11 & 4.45 & 16.70 \\
QD390 & $\mathrm{C}_{21} \mathrm{H}_{13} \mathrm{~N}_{3} \mathrm{O}_{2}$ & 74.33 & 3.86 & 12.38 & 74.22 & 3.89 & 12.62 \\
QD391 & $\mathrm{C}_{21} \mathrm{H}_{13} \mathrm{~N}_{3} \mathrm{O}_{2}$ & 74.33 & 3.86 & 12.38 & 74.46 & 3.90 & 12.02 \\
QD392 & $\mathrm{C}_{22} \mathrm{H}_{17} \mathrm{~N}_{3} \mathrm{O}_{2}$ & 74.35 & 4.82 & 11.82 & 74.18 & 5.07 & 11.77 \\
QD393 & $\mathrm{C}_{20} \mathrm{H}_{19} \mathrm{~N}_{3} \mathrm{O}_{2}$ & 72.05 & 5.74 & 12.60 & 72.15 & 5.97 & 12.51 \\
QD394 & $\mathrm{C}_{19} \mathrm{H}_{19} \mathrm{~N}_{5} \mathrm{O}_{2}$ & 65.32 & 5.48 & 20.04 & 65.03 & 5.78 & 19.88 \\
QD395 & $\mathrm{C}_{19} \mathrm{H}_{12} \mathrm{~N}_{4} \mathrm{O}_{2}$ & 69.51 & 3.68 & 17.06 & 69.77 & 3.84 & 16.88 \\
QD394-Me & $\mathrm{C}_{20} \mathrm{H}_{21} \mathrm{~N}_{5} \mathrm{O}_{2}$ & 66.10 & 5.82 & 19.07 & 66.28 & 5.69 & 19.41 \\
(QD430) & & & & & & & \\
\hline
\end{tabular}


Table S2. Molecular formula strings of compounds.

\begin{tabular}{|c|c|}
\hline Compound ID & SMILES \\
\hline 1 & $\mathrm{O}=\mathrm{C} 1 \mathrm{C}=\mathrm{CC}(\mathrm{C} 2=\mathrm{C} 1 \mathrm{C}=\mathrm{NC}=\mathrm{N} 2)=\mathrm{O}$ \\
\hline 2 & $\mathrm{COC} 1=\mathrm{CC}=\mathrm{C}(\mathrm{OC}) \mathrm{C}=\mathrm{C} 1 \mathrm{C}([\mathrm{H}])=\mathrm{O}$ \\
\hline 3 & $\mathrm{COC} 1=\mathrm{CC}=\mathrm{C}(\mathrm{OC}) \mathrm{C}([\mathrm{N}+]([\mathrm{O}-])=\mathrm{O})=\mathrm{C} 1 \mathrm{C}([\mathrm{H}])=\mathrm{O}$ \\
\hline 4 & $\mathrm{COC} 1=\mathrm{CC}=\mathrm{C}(\mathrm{OC}) \mathrm{C}(\mathrm{C}(\mathrm{NC}([\mathrm{H}])=\mathrm{O}) \mathrm{NC}([\mathrm{H}])=\mathrm{O})=\mathrm{C} 1[\mathrm{~N}+]([\mathrm{O}-])=\mathrm{O}$ \\
\hline 5 & $\mathrm{COC} 1=\mathrm{CC}=\mathrm{C}(\mathrm{OC}) \mathrm{C} 2=\mathrm{C} 1 \mathrm{~N}=\mathrm{CN}=\mathrm{C} 2$ \\
\hline 6 & $\mathrm{COC} 1=\mathrm{CC}(\mathrm{C})=\mathrm{C}(\mathrm{OC}) \mathrm{C}=\mathrm{C} 1[\mathrm{~N}+]([\mathrm{O}-])=\mathrm{O}$ \\
\hline 7 & $\mathrm{COC} 1=\mathrm{CC}(\mathrm{C})=\mathrm{C}(\mathrm{OC}) \mathrm{C}([\mathrm{N}+]([\mathrm{O}-])=\mathrm{O})=\mathrm{C} 1 \mathrm{C}([\mathrm{H}])=\mathrm{O}$ \\
\hline 8 & $\mathrm{COC} 1=\mathrm{CC}(\mathrm{C})=\mathrm{C}(\mathrm{OC}) \mathrm{C}([\mathrm{N}+]([\mathrm{O}-])=\mathrm{O})=\mathrm{C} 1 \mathrm{C}(\mathrm{NC}([\mathrm{H}])=\mathrm{O}) \mathrm{NC}([\mathrm{H}])=\mathrm{O}$ \\
\hline 9 & $\mathrm{COC} 1=\mathrm{CC}(\mathrm{C})=\mathrm{C}(\mathrm{OC}) \mathrm{C} 2=\mathrm{C} 1 \mathrm{C}=\mathrm{NC}=\mathrm{N} 2$ \\
\hline 10 & $\mathrm{O}=\mathrm{C}(\mathrm{C} 1=\mathrm{C} 2 \mathrm{C}=\mathrm{NC}=\mathrm{N} 1) \mathrm{C}(\mathrm{C})=\mathrm{CC} 2=\mathrm{O}$ \\
\hline 11 & $\mathrm{COC} 1=\mathrm{CC}(\mathrm{C})=\mathrm{C}(\mathrm{OC}) \mathrm{C}=\mathrm{C} 1 \mathrm{~N}$ \\
\hline 12 & $\mathrm{COC} 1=\mathrm{CC}(\mathrm{C})=\mathrm{C}(\mathrm{OC}) \mathrm{C}=\mathrm{C} 1 \mathrm{NC}(\mathrm{OCC})=\mathrm{O}$ \\
\hline 13 & $\mathrm{COC} 1=\mathrm{CC}(\mathrm{C})=\mathrm{C}(\mathrm{OC}) \mathrm{C} 2=\mathrm{C} 1 \mathrm{~N}(\mathrm{CN}=\mathrm{C} 2) \mathrm{C}(\mathrm{OCC})=\mathrm{O}$ \\
\hline 14 & $\mathrm{COC} 1=\mathrm{CC}(\mathrm{C})=\mathrm{C}(\mathrm{OC}) \mathrm{C} 2=\mathrm{C} 1 \mathrm{~N}=\mathrm{CN}=\mathrm{C} 2$ \\
\hline 15 & $\mathrm{O}=\mathrm{C}(\mathrm{C} 1=\mathrm{C} 2 \mathrm{C}=\mathrm{NC}=\mathrm{N} 1) \mathrm{C}=\mathrm{C}(\mathrm{C}) \mathrm{C} 2=\mathrm{O}$ \\
\hline QD385 & $\mathrm{O}=\mathrm{C}(\mathrm{C} 1=\mathrm{C} 2 \mathrm{~N}=\mathrm{CN}=\mathrm{C} 1) \mathrm{C}(\mathrm{NC} 3=\mathrm{CC}=\mathrm{CC}(\mathrm{C} 4=\mathrm{CC}=\mathrm{CC}=\mathrm{C} 4)=\mathrm{C} 3)=\mathrm{CC} 2=\mathrm{O}$ \\
\hline QD386 & $\mathrm{O}=\mathrm{C}(\mathrm{C} 1=\mathrm{C} 2 \mathrm{~N}=\mathrm{CN}=\mathrm{C} 1) \mathrm{C}(\mathrm{NC} 3=\mathrm{CC}=\mathrm{C}(\mathrm{C} 4=\mathrm{CC}=\mathrm{C}(\mathrm{Cl}) \mathrm{C}=\mathrm{C} 4) \mathrm{C}=\mathrm{C} 3)=\mathrm{CC} 2=\mathrm{O}$ \\
\hline QD387 & $\mathrm{O}=\mathrm{C}(\mathrm{C} 1=\mathrm{C} 2 \mathrm{~N}=\mathrm{CN}=\mathrm{C} 1) \mathrm{C}(\mathrm{NC} 3=\mathrm{CC}=\mathrm{C}(\mathrm{C} 4=\mathrm{CC}(\mathrm{Cl})=\mathrm{CC}(\mathrm{Cl})=\mathrm{C} 4) \mathrm{C}=\mathrm{C} 3)=\mathrm{CC} 2=\mathrm{O}$ \\
\hline QD388 & $\mathrm{O}=\mathrm{C}(\mathrm{C} 1=\mathrm{C} 2 \mathrm{~N}=\mathrm{CN}=\mathrm{C} 1) \mathrm{C}(\mathrm{NC} 3=\mathrm{CC}=\mathrm{C}(\mathrm{N} 4 \mathrm{CCCCC} 4) \mathrm{C}=\mathrm{C} 3)=\mathrm{CC} 2=\mathrm{O}$ \\
\hline QD389 & $\mathrm{O}=\mathrm{C}(\mathrm{C} 1=\mathrm{C} 2 \mathrm{~N}=\mathrm{CN}=\mathrm{C} 1) \mathrm{C}(\mathrm{NC} 3=\mathrm{CC}=\mathrm{C}(\mathrm{N} 4 \mathrm{CCOCC} 4) \mathrm{C}=\mathrm{C} 3)=\mathrm{CC} 2=\mathrm{O}$ \\
\hline QD390 & $\mathrm{O}=\mathrm{C}(\mathrm{C} 1=\mathrm{C} 2 \mathrm{~N}=\mathrm{CN}=\mathrm{C} 1) \mathrm{C}(\mathrm{NC} 3=\mathrm{C} 4 \mathrm{C}(\mathrm{C}(\mathrm{C}=\mathrm{CC}=\mathrm{C} 5)=\mathrm{C} 5 \mathrm{C} 4)=\mathrm{CC}=\mathrm{C} 3)=\mathrm{CC} 2=\mathrm{O}$ \\
\hline QD391 & $\mathrm{O}=\mathrm{C}(\mathrm{C} 1=\mathrm{C} 2 \mathrm{~N}=\mathrm{CN}=\mathrm{C} 1) \mathrm{C}(\mathrm{NC} 3=\mathrm{CC}=\mathrm{C}(\mathrm{C}(\mathrm{C}=\mathrm{CC}=\mathrm{C} 4)=\mathrm{C} 4 \mathrm{C} 5) \mathrm{C} 5=\mathrm{C} 3)=\mathrm{CC} 2=\mathrm{O}$ \\
\hline QD392 & $\mathrm{O}=\mathrm{C}(\mathrm{C} 1=\mathrm{C} 2 \mathrm{~N}=\mathrm{CN}=\mathrm{C} 1) \mathrm{C}(\mathrm{NC} 3=\mathrm{CC}=\mathrm{C}(\mathrm{C} 4=\mathrm{CC}=\mathrm{C}(\mathrm{CC}) \mathrm{C}=\mathrm{C} 4) \mathrm{C}=\mathrm{C} 3)=\mathrm{CC} 2=\mathrm{O}$ \\
\hline QD393 & $\mathrm{O}=\mathrm{C}(\mathrm{C} 1=\mathrm{C} 2 \mathrm{~N}=\mathrm{CN}=\mathrm{C} 1) \mathrm{C}(\mathrm{NC} 3=\mathrm{CC}=\mathrm{C}(\mathrm{C} 4 \mathrm{CCCCC} 4) \mathrm{C}=\mathrm{C} 3)=\mathrm{CC} 2=\mathrm{O}$ \\
\hline QD394 & $\mathrm{O}=\mathrm{C}(\mathrm{C} 1=\mathrm{C} 2 \mathrm{~N}=\mathrm{CN}=\mathrm{C} 1) \mathrm{C}(\mathrm{NC} 3=\mathrm{CC}=\mathrm{C}(\mathrm{N} 4 \mathrm{CCN}(\mathrm{C}) \mathrm{CC} 4) \mathrm{C}=\mathrm{C} 3)=\mathrm{CC} 2=\mathrm{O}$ \\
\hline QD395 & $\mathrm{O}=\mathrm{C}(\mathrm{C} 1=\mathrm{C} 2 \mathrm{~N}=\mathrm{CN}=\mathrm{C} 1) \mathrm{C}(\mathrm{NC} 3=\mathrm{CC}=\mathrm{C}(\mathrm{C} 4=\mathrm{CC}=\mathrm{CC}=\mathrm{N} 4) \mathrm{C}=\mathrm{C} 3)=\mathrm{CC} 2=\mathrm{O}$ \\
\hline \multicolumn{2}{|l|}{ QD394-Me } \\
\hline$(\mathrm{QD} 430)$ & $\mathrm{O}=\mathrm{C}(\mathrm{C} 1=\mathrm{C} 2 \mathrm{~N}=\mathrm{CN}=\mathrm{C} 1) \mathrm{C}(\mathrm{NC} 3=\mathrm{CC}=\mathrm{C}(\mathrm{N} 4 \mathrm{CCN}(\mathrm{C}) \mathrm{CC} 4) \mathrm{C}=\mathrm{C} 3)=\mathrm{C}(\mathrm{C}) \mathrm{C} 2=\mathrm{O}$ \\
\hline
\end{tabular}

Table S3. Top 25 upregulated (A) and downregulated (B) enriched C2 gene sets in QD394-treated MIA PaCa-2 cells. FDR $<0.001$.

(A)

\begin{tabular}{llll}
\hline NAME & SIZE & NES & FDR \\
\hline NAGASHIMA_EGF_SIGNALING_UP & 53 & 3.14 & 0 \\
NAGASHIMA_NRG1_SIGNALING_UP & 149 & 3.09 & 0 \\
SABATES_COLORECTAL_ADENOMA_DN & 153 & 3.08 & 0 \\
BENPORATH_PRC2_TARGETS & 341 & 3.01 & 0 \\
KEGG_NEUROACTIVE_LIGAND_RECEPTOR_INTERA & & & \\
CTION & 130 & 2.94 & 0
\end{tabular}




\begin{tabular}{llll} 
UZONYI_RESPONSE_TO_LEUKOTRIENE_AND_THRO & & & \\
MBIN & 31 & 2.83 & 0 \\
BURTON_ADIPOGENESIS_PEAK_AT_2HR & 41 & 2.82 & 0 \\
KONDO_PROSTATE_CANCER_WITH_H3K27ME3 & 93 & 2.80 & 0 \\
MIKKELSEN_MEF_HCP_WITH_H3K27ME3 & 280 & 2.77 & 0 \\
REACTOME_GPCR_LIGAND_BINDING & 147 & 2.76 & 0 \\
GERY_CEBP_TARGETS & 100 & 2.74 & 0 \\
HELLER_SILENCED_BY_METHYLATION_DN & 79 & 2.73 & 0 \\
GESERICK_TERT_TARGETS_DN & 19 & 2.70 & 0 \\
KEGG_SYSTEMIC_LUPUS_ERYTHEMATOSUS & 81 & 2.69 & 0 \\
WALLACE_PROSTATE_CANCER_RACE_UP & 151 & 2.68 & 0 \\
SCHLESINGER_METHYLATED_DE_NOVO_IN_CANCE & & & \\
R & 52 & 2.67 & 0 \\
NOJIMA_SFRP2_TARGETS_UP & 25 & 2.66 & 0 \\
SATO_SILENCED_BY_DEACETYLATION_IN_PANCRE & & & \\
ATIC_CANCER & 34 & 2.66 & 0 \\
PODAR_RESPONSE_TO_ADAPHOSTIN_UP & 121 & 2.65 & 0 \\
MEISSNER_NPC_HCP_WITH_H3K4ME2_AND_H3K27M & & & \\
E3 & 185 & 2.65 & 0 \\
REACTOME_CLASS_A1_RHODOPSIN_LIKE_RECEPTO & & & \\
RS & 93 & 2.65 & 0 \\
MIKKELSEN_NPC_HCP_WITH_H3K4ME3_AND_H3K27 & & & \\
ME3 & 121 & 2.62 & 0 \\
KIM_RESPONSE_TO_TSA_AND_DECITABINE_UP & 82 & 2.62 & 0 \\
NABA_CORE_MATRISOME & 173 & 2.62 & 0 \\
NABA_MATRISOME_ASSOCIATED & 368 & 2.59 & 0 \\
\hline (B) & & & \\
\hline
\end{tabular}

(B)

\begin{tabular}{llll}
\hline NAME & SIZE & NES & FDR \\
\hline DACOSTA_UV_RESPONSE_VIA_ERCC3_COMMON & & & \\
_DN & 449 & -4.82 & 0 \\
SHEN_SMARCA2_TARGETS_UP & 376 & -3.97 & 0 \\
MARTINEZ_RESPONSE_TO_TRABECTEDIN & 41 & -3.72 & 0 \\
MITSIADES_RESPONSE_TO_APLIDIN_DN & 209 & -3.63 & 0 \\
ZHANG_TLX_TARGETS_36HR_DN & 167 & -3.53 & 0 \\
DAZARD_RESPONSE_TO_UV_NHEK_DN & 274 & -3.50 & 0 \\
DAZARD_UV_RESPONSE_CLUSTER_G6 & 135 & -3.27 & 0 \\
GABRIELY_MIR21_TARGETS & 243 & -3.27 & 0 \\
BIDUS_METASTASIS_UP & 174 & -3.24 & 0 \\
TOYOTA_TARGETS_OF_MIR34B_AND_MIR34C & 338 & -3.23 & 0 \\
PUJANA_BRCA_CENTERED_NETWORK & 105 & -3.15 & 0 \\
MILI_PSEUDOPODIA_HAPTOTAXIS_UP & 420 & -3.11 & 0
\end{tabular}




\begin{tabular}{|c|c|c|c|}
\hline UMBER & 89 & -3.10 & 0 \\
\hline PUJANA_XPRSS_INT_NETWORK & 145 & -3.08 & 0 \\
\hline DACOSTA_UV RESPONSE_VIA_ERCC 3 XPCS_DN & 72 & -2.93 & 0 \\
\hline KEGG UBIQUITIN MEDIATED PROTEOLYSIS & 111 & -2.89 & 0 \\
\hline DACOSTA_UV_RESPONSE_VIA_ERCC3_TTD_DN & 67 & -2.88 & 0 \\
\hline HAMAI_APOPTOSIS_VIA_TRAIL_UP & 480 & -2.88 & 0 \\
\hline JOHNSTONE PARVB TARGETS 2 DN & 280 & -2.86 & 0 \\
\hline ZHANG_BREAST_CANCER_PROGENITORS_UP & 343 & -2.84 & 0 \\
\hline ZHANG_TLX_TARGETS_DN & 79 & -2.83 & 0 \\
\hline PUJANA_BRCA2_PCC_NETWORK & 337 & -2.81 & 0 \\
\hline ENK UV RESPONSE KERATINOCYTE DN & 426 & -2.79 & 0 \\
\hline REACTOME_MITOTIC_PROMETAPHASE & 68 & -2.77 & 0 \\
\hline RAMALHO_STEMNESS_UP & 165 & -2.69 & $2.49 \mathrm{E}-05$ \\
\hline
\end{tabular}

Table S4. STRING analysis of 17 upregulated genes/proteins in common between Bru-seq and proteomics. Biological process GO terms are ranked by FDR.

\begin{tabular}{|c|c|c|c|}
\hline Biological Process (GO) & Count & FDR & Matching proteins \\
\hline $\begin{array}{l}\text { response to endoplasmic reticulum } \\
\text { stress }\end{array}$ & 5 & 0.0018 & $\begin{array}{l}\text { ASNS,CTH,HSPA5,PPP1R15A, } \\
\text { SESN2 }\end{array}$ \\
\hline cellular response to glucose starvation & 3 & 0.003 & ASNS,HSPA5,SESN2 \\
\hline $\begin{array}{l}\text { negative regulation of response to } \\
\text { stimulus }\end{array}$ & 8 & 0.0055 & $\begin{array}{l}\text { CD55,CTH,HMOX1,HSPA5, } \\
\text { PPP1R15A,RIOK3,SERPINE1, } \\
\text { SESN2 }\end{array}$ \\
\hline $\begin{array}{l}\text { negative regulation of immune effector } \\
\text { process }\end{array}$ & 3 & 0.0078 & CD55,HMOX1,RIOK3 \\
\hline response to stress & 10 & 0.0078 & $\begin{array}{l}\text { ASNS,CD55,CTH,HMOX1, } \\
\text { HSPA5,IFRD1,PPP1R15A, } \\
\text { RIOK3, SERPINE1,SESN2 }\end{array}$ \\
\hline $\begin{array}{l}\text { negative regulation of signal } \\
\text { transduction }\end{array}$ & 7 & 0.0078 & $\begin{array}{l}\text { CTH,HMOX1,HSPA5, } \\
\text { PPP1R15A,RIOK3,SERPINE1, } \\
\text { SESN2 }\end{array}$ \\
\hline $\begin{array}{l}\text { endoplasmic reticulum unfolded } \\
\text { protein response }\end{array}$ & 3 & 0.0078 & ASNS,CTH,HSPA5 \\
\hline cellular response to nutrient levels & 4 & 0.0078 & ASNS,HMOX1,HSPA5,SESN2 \\
\hline $\begin{array}{l}\text { PERK-mediated unfolded protein } \\
\text { response }\end{array}$ & 2 & 0.0078 & ASNS,HSPA5 \\
\hline $\begin{array}{l}\text { negative regulation of apoptotic } \\
\text { process }\end{array}$ & 6 & 0.0078 & $\begin{array}{l}\text { ASNS,CBX4,CTH,HMOX1, } \\
\text { HSPA5,SERPINE1 }\end{array}$ \\
\hline regulation of translation in response to & 2 & 0.0078 & PPP1R15A,SESN2 \\
\hline
\end{tabular}


stress

regulation of transcription from RNA

3

0.0078

HMOX1,HSPA5,SESN2

polymerase II promoter in response to

stress

regulation of transcription from RNA

2

0.0078 HMOX1,SESN2

polymerase II promoter in response to

oxidative stress

negative regulation of cellular process

$12 \quad 0.0078$

ASNS,CBX4,CD55,CTH, HMOX1,HSPA5,IFRD1,JUND, PPP1R15A,RIOK3,SERPINE1, SESN2

cellular response to chemical stimulus

9

0.0078

ASNS,CTH,HMOX1,HSPA5, JUND,RIOK3,SERPINE1, SESN2,ZFAND2A

cellular response to arsenic-containing 2

2

0.0078

HMOX1,ZFAND2A

negative regulation of endoplasmic

2

0.0078

HSPA5,PPP1R15A

reticulum unfolded protein response

regulation of PERK-mediated

2

unfolded protein response

aspartate family amino acid

biosynthetic process

response to organic substance

$0.0078 \quad$ HSPA5,PPP1R15A

$2 \quad 0.0079 \quad$ ASNS,CTH

$9 \quad 0.0082 \quad$ ASNS,CTH,HMOX1,HSPA5, JUND,PLIN2,RIOK3, SERPINE1,SESN2

Table S5. STRING analysis of 35 downregulated genes/proteins in common between Bru-seq and proteomics. Biological process GO terms are ranked by FDR. PNPT1 and LRPPRC are labeled in red.

\begin{tabular}{|c|c|c|c|}
\hline Biological Process (GO) & Count & FDR & Matching proteins \\
\hline $\begin{array}{l}\text { mitochondrial gene } \\
\text { expression }\end{array}$ & 8 & $1.04 \mathrm{E}-07$ & $\begin{array}{l}\text { MRPL19,MRPS25,MRPS27,MRPS35,MRPS9, } \\
\text { PNPT1,PTCD3,TSFM }\end{array}$ \\
\hline $\begin{array}{l}\text { mitochondrial } \\
\text { translational elongation }\end{array}$ & 7 & $1.16 \mathrm{E}-07$ & $\begin{array}{l}\text { MRPL19,MRPS25,MRPS27,MRPS35,MRPS9, } \\
\text { PTCD3,TSFM }\end{array}$ \\
\hline $\begin{array}{l}\text { mitochondrial } \\
\text { translational termination }\end{array}$ & 6 & $2.58 \mathrm{E}-06$ & $\begin{array}{l}\text { MRPL19,MRPS25,MRPS27,MRPS35,MRPS9, } \\
\text { PTCD3 }\end{array}$ \\
\hline $\begin{array}{l}\text { regulation of } \\
\text { mitochondrial translation }\end{array}$ & 4 & $1.74 \mathrm{E}-05$ & FASTKD2,LRPPRC,MRPS27,TSFM \\
\hline cellular respiration & 6 & $2.73 \mathrm{E}-05$ & $\begin{array}{l}\text { ACO2,FASTKD2,NDUFS1,NDUFS4,SDHC, } \\
\text { SUCLG1 }\end{array}$ \\
\hline oxidation-reduction & 10 & 0.0002 & ACO2,AUH,FASTKD2,HADHA,NDUFS1, \\
\hline
\end{tabular}




\begin{tabular}{|c|c|c|c|}
\hline process & & & NDUFS4,PEX13,SDHC,SUCLG1,TYW1 \\
\hline $\begin{array}{l}\text { generation of precursor } \\
\text { metabolites and energy }\end{array}$ & 7 & 0.00026 & $\begin{array}{l}\text { ACO2,FASTKD2,FECH,NDUFS1,NDUFS4, } \\
\text { SDHC,SUCLG1 }\end{array}$ \\
\hline metabolic process & 30 & 0.00029 & $\begin{array}{l}\text { ACO2,AUH,FAM120B,FAM20B,FASTKD2, } \\
\text { FECH,HADHA,LRPPRC,MCCC1,MRPL19, } \\
\text { MRPS25,MRPS27,MRPS35,MRPS9,NCOA6, } \\
\text { NDUFS1,NDUFS4,NFU1,PEX13,PNPT1, } \\
\text { PTCD3,SATB1,SDHC,SPG7,SUCLG1, } \\
\text { TFAP2A,TSFM,TYW1,XPNPEP3,ZNF318 }\end{array}$ \\
\hline peptide metabolic process & 7 & 0.001 & $\begin{array}{l}\text { MRPL19,MRPS25,MRPS27,MRPS35,MRPS9, } \\
\text { PTCD3,TSFM }\end{array}$ \\
\hline $\begin{array}{l}\text { cellular metabolic } \\
\text { process }\end{array}$ & 28 & 0.001 & $\begin{array}{l}\text { ACO2,AUH,FAM120B,FAM20B,FASTKD2, } \\
\text { FECH,HADHA,LRPPRC,MCCC1,MRPL19, } \\
\text { MRPS25,MRPS27,MRPS35,MRPS9,NCOA6, } \\
\text { NDUFS1,NDUFS4,NFU1,PEX13,PNPT1, } \\
\text { PTCD3,SATB1,SDHC,SUCLG1,TFAP2A, } \\
\text { TSFM,TYW1,ZNF318 }\end{array}$ \\
\hline tricarboxylic acid cycle & 3 & 0.0012 & ACO2,SDHC,SUCLG1 \\
\hline $\begin{array}{l}\text { cellular amide metabolic } \\
\text { process }\end{array}$ & 8 & 0.0012 & $\begin{array}{l}\text { MCCC1,MRPL19,MRPS25,MRPS27, } \\
\text { MRPS35,MRPS9,PTCD3,TSFM }\end{array}$ \\
\hline $\begin{array}{l}\text { protein-containing } \\
\text { complex subunit } \\
\text { organization }\end{array}$ & 12 & 0.0012 & $\begin{array}{l}\text { ATPAF1,FASTKD2,MCCC1,MRPL19, } \\
\text { MRPS25,MRPS27,MRPS35,MRPS9,NDUFS1, } \\
\text { NDUFS4,PNPT1,PTCD3 }\end{array}$ \\
\hline $\begin{array}{l}\text { regulation of } \\
\text { mitochondrial RNA } \\
\text { catabolic process }\end{array}$ & 2 & 0.0014 & LRPPRC,PNPT1 \\
\hline citrate metabolic process & 3 & 0.0014 & ACO2,SDHC,SUCLG1 \\
\hline leucine catabolic process & 2 & 0.0016 & AUH,MCCC1 \\
\hline $\begin{array}{l}\text { organic substance } \\
\text { metabolic process }\end{array}$ & 28 & 0.0016 & $\begin{array}{l}\text { ACO2,AUH,FAM120B,FASTKD2,FECH, } \\
\text { HADHA,LRPPRC,MCCC1,MRPL19, } \\
\text { MRPS25,MRPS27,MRPS35,MRPS9,NCOA6, } \\
\text { NDUFS1,NDUFS4,PEX13,PNPT1,PTCD3, } \\
\text { SATB1,SDHC,SPG7,SUCLG1,TFAP2A, } \\
\text { TSFM,TYW1,XPNPEP3,ZNF318 }\end{array}$ \\
\hline leucine metabolic process & 2 & 0.0026 & $\mathrm{AUH}, \mathrm{MCCC} 1$ \\
\hline $\begin{array}{l}\text { cellular nitrogen } \\
\text { compound metabolic } \\
\text { process }\end{array}$ & 20 & 0.0026 & $\begin{array}{l}\text { FAM120B,FECH,LRPPRC,MCCC1,MRPL19, } \\
\text { MRPS25,MRPS27,MRPS35,MRPS9,NCOA6, } \\
\text { NDUFS1,NDUFS4,PNPT1,PTCD3,SATB1, } \\
\text { SDHC,TFAP2A,TSFM,TYW1,ZNF318 }\end{array}$ \\
\hline
\end{tabular}


Table S6. Tissue distribution of QD394-Me in mouse. IV, $10 \mathrm{mg} / \mathrm{kg} ;$ PO, $20 \mathrm{mg} / \mathrm{kg}, 1 \mathrm{~h}$.

\begin{tabular}{lll}
\hline \multirow{2}{*}{$1 \mathrm{~h}$} & \multicolumn{2}{l}{ Concentrations $(\mathrm{ng} / \mathrm{mL}$ or $\mathrm{ng} / \mathrm{g})$} \\
& PO $20 \mathrm{mg} / \mathrm{kg}$ & IV $10 \mathrm{mg} / \mathrm{kg}$ \\
\hline Plasma & 52 & 25.8 \\
Blood & 209 & 86.4 \\
Skin & 33.1 & 20.45 \\
Bone & 13.7 & 36.5 \\
Lung & 22.6 & 35.9 \\
Pancreas & 28.4 & 55 \\
Spleen & 40 & 249.5 \\
Kidney & 126.5 & 142.5 \\
Colon & 339 & 277.5 \\
Liver & 51 & 31.15 \\
\hline
\end{tabular}

Table S7. Cytotoxicity of QD394, QD394-Me, and napabucasin in GPX4-knockdown PANC-1 and BxPC-3 cells. siNC: samples treated with scramble siRNA; siGPX4: samples treated with GPX4 siRNA.

\begin{tabular}{lllll}
\hline \multirow{2}{*}{$\mathrm{IC}_{50}(\mu \mathrm{M})$} & \multicolumn{2}{c}{ PANC-1 } & \multicolumn{2}{c}{ BxPC-3 } \\
& siNC & siGPX4 & siNC & siGPX4 \\
\hline QD394 & 0.362 & 1.064 & 0.715 & 1.292 \\
QD394-Me & 2.734 & 6.74 & 3.55 & 4.649 \\
Napa & 1.159 & 3.143 & 1.041 & 1.72 \\
\hline
\end{tabular}

Table S8. Top upregulated (A) and downregulated (B) enriched hallmark gene sets in QD394-Me-treated MIA PaCa-2 cells. FDR $<0.05$.

(A)

\begin{tabular}{llll}
\hline NAME & SIZE & NES & FDR \\
\hline HALLMARK_MTORC1_SIGNALING & 186 & 2.51 & 0 \\
HALLMARK_TNFA_SIGNALING_VIA_NFKB & 160 & 2.42 & 0 \\
HALLMARK_APOPTOSIS & 126 & 2.23 & 0 \\
HALLMARK_UNFOLDED_PROTEIN_RESPONSE & 107 & 2.20 & 0 \\
HALLMARK_PROTEIN_SECRETION & 92 & 2.18 & 0 \\
HALLMARK_INFLAMMATORY_RESPONSE & 98 & 2.08 & 0 \\
HALLMARK_ANDROGEN_RESPONSE & 88 & 2.06 & 0
\end{tabular}




\begin{tabular}{|c|c|c|c|}
\hline \multirow{2}{*}{ HALLMARK_MYC_TARGETS_V1 } & 197 & 1.99 & 0 \\
\hline & 119 & 1.90 & 0 \\
\hline HALLMARK_P53_PATHWAY & 172 & 1.90 & 0 \\
\hline HALLMARK_HYP̄OXIA & 154 & 1.86 & 0 \\
\hline HALLMARK_INTERFERON_GAMMA_RESPONSE & 132 & 1.77 & 0.001 \\
\hline HALLMARK_HEME_METABOLISM & 156 & 1.76 & 0.002 \\
\hline $\begin{array}{l}\text { HALLMARK_REACTIVE_OXYGEN_SPECIES } \\
\text { PATHWAY }\end{array}$ & 43 & 1.72 & 0.003 \\
\hline HALLMARK COMPLEMENT & 124 & 1.70 & 0.003 \\
\hline HALLMARK_ALLOGRAFT_REJECTION & 92 & 1.68 & 0.004 \\
\hline HALLMARK_CHOLESTEROL_HOMEOSTASIS & 65 & 1.64 & 0.006 \\
\hline HALLMARK_XENOBIOTIC_METABOLISM & 140 & 1.62 & 0.007 \\
\hline HALLMARK_KRAS_SIGNALING_UP & 108 & 1.61 & 0.008 \\
\hline HALLMARK GLYCOLYSIS & 171 & 1.56 & 0.013 \\
\hline HALLMARK_IL6_JAK_STAT3_SIGNALING & 48 & 1.52 & 0.021 \\
\hline HALLMARK_FATTY_ACID_METABOLISM & 127 & 1.51 & 0.021 \\
\hline $\begin{array}{l}\text { HALLMARK_EPITHELIAL_MESENCHYMAL_ } \\
\text { TRANSITION }\end{array}$ & 123 & 1.45 & 0.040 \\
\hline HALLMARK_UV_RESPONSE_UP & 135 & 1.43 & 0.043 \\
\hline HALLMARK G2M CHECKPOINT & 195 & 1.43 & 0.041 \\
\hline HALLMARK_IL2_STAT5_SIGNALING & 136 & 1.43 & 0.042 \\
\hline \multicolumn{4}{|c|}{ (B) } \\
\hline NAME & \multicolumn{2}{|r|}{ NES } & FDR \\
\hline HALLMARK_APICAL_JUNCTION & \multicolumn{2}{|r|}{-1.78} & 0.007 \\
\hline HALLMARK MYOGENESIS & \multicolumn{2}{|r|}{-1.71} & 0.010 \\
\hline HALLMARK KRAS SIGNALING DN & \multicolumn{2}{|r|}{-1.68} & 0.007 \\
\hline
\end{tabular}

Table S9. Top upregulated enriched KEGG gene sets in QD394-Me-treated MIA PaCa-2 cells. No downregulated enriched KEGG gene sets were significant. $\mathrm{FDR}<0.05$.

\begin{tabular}{|c|c|c|c|}
\hline NAME & SIZE & NES & FDR \\
\hline KEGG_SYSTEMIC_LUPUS_ERYTHEMATOSUS & 69 & 2.51 & 0 \\
\hline KEGG_RIBOSOME & 83 & 2.42 & 0 \\
\hline KEGG_PROTEIN_EXPORT & 23 & 2.23 & 0.002 \\
\hline KEGG_PROTEASOME & 38 & 2.20 & 0.017 \\
\hline $\begin{array}{l}\text { KEGG_NOD_LIKE_RECEPTOR_SIGNALING_ } \\
\text { PATHWAY }\end{array}$ & 43 & 2.18 & 0.032 \\
\hline $\begin{array}{l}\text { KEGG_PORPHYRIN_AND_CHLOROPHYLL_ } \\
\text { METABOLISM }\end{array}$ & 22 & 2.08 & 0.030 \\
\hline
\end{tabular}

